\title{
AUTOMATIC BUILDING EXTRACTION AND ROOF RECONSTRUCTION IN 3K IMAGERY BASED ON LINE SEGMENTS
}

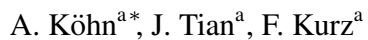 \\ ${ }^{a}$ German Aerospace Center (DLR), Remote Sensing Technology Institute (IMF), 82234 Weßling, Germany - \\ (alexander.koehn, jiaojiao.tian, franz.kurz)@dlr.de
}

Commission III, WG III/4

KEY WORDS: Building detection, line segment detection, DSM, morphological filtering, RANSAC, plane fitting, vegetation identification

\begin{abstract}
:
We propose an image processing workflow to extract rectangular building footprints using georeferenced stereo-imagery and a derivative digital surface model (DSM) product. The approach applies a line segment detection procedure to the imagery and subsequently verifies identified line segments individually to create a footprint on the basis of the DSM. The footprint is further optimized by morphological filtering. Towards the realization of 3D models, we decompose the produced footprint and generate a 3D point cloud from DSM height information. By utilizing the robust RANSAC plane fitting algorithm, the roof structure can be correctly reconstructed. In an experimental part, the proposed approach has been performed on $3 \mathrm{~K}$ aerial imagery.
\end{abstract}

\section{INTRODUCTION}

Automatic building extraction and reconstruction from very high resolution remote sensing images are difficult tasks. The detection accuracy strongly depends on image quality and building shapes. In addition to $2 \mathrm{D}$ information from spectral and panchromatic images, height information from digital surface model (DSM) has received increasingly attention for automatic building extraction.

A considerable amount of studies addresses DSM-assisted building footprint extraction. Some of them fuse data from different sensors, chiefly multispectral images and LiDAR-derived DSMs (Matikainen et al., 2010; Hermosilla et al., 2011; Grigillo and Kanjir, 2012). Those studies, however, rely on different data sources which may imply difficulties concerning the availability and temporal coincidence of the data. Exploiting the potentials of a single platform could yield a solution. Hence, a number of authors directly extracted footprint shapes from LiDAR point clouds (Wang et al., 2006; Zhang et al., 2006; Arefi et al., 2008) or nadir RGB imagery (Shorter and Kasparis, 2009). Stereo images also provide possibilities for detecting building geometries using optical and height information derived from the same data source (Arefi and Reinartz, 2013; Tian et al., 2014) or solely height information (Weidner, 1997). Photogrammetric techniques have been used to extract three-dimensional line segments for 3D model generation (Zebedin et al., 2008). These line segments, however, require a good perspective coverage of the scene in order to be useful for building extraction. We therefore studied the potentials and limitations of extracting line segments from individual images used for DSM generation and subsequently verifying them on the basis of the DSM.

A major constraint of directly inferring building footprints from DSM data is the presence of vegetation. Several approaches address this issue, e.g. by applying a Normalized Difference Vegetation Index (NDVI) mask to the image (Grigillo and Kanjir, 2012), computing the variance of surface normal vectors within the DSM (Weidner, 1997) or segmenting a RGB image and then

${ }^{*}$ Corresponding author removing color-invariant segments indicating vegetation (Shorter and Kasparis, 2009). However, spectral identification of vegetation is not reliable when an infrared channel is missing, especially when vegetation is (partially) cast over by shadows from adjacent structures. It is further compromised when the acquisition time of the images does not coincide with the growing season. On the other hand, the variance of surface normals may not be a reliable indicator for vegetation if there is noise present in the DSM (see section 3.1). Therefore, another geometric criteria is proposed by applying a line segment detection algorithm to the aerial image and a standard deviation threshold to the underlying DSM. In contrast to man-made structures, line segments detected in canopies are expected to be less directional.

In a last step of building reconstruction, the detected building footprints are segmented to obtain simpler building geometries. A RANSAC-based plane fitting procedure is applied to the pixels in each segment by which 3D building roofs are reconstructed.

\section{METHODOLOGY}

The methodology is divided into four sections. We will first describe the segmentation of the whole scene to obtain image subsets of individual buildings. Line segments are then identified and filtered to derive a raw building footprint. In an optimization step, morphological filtering is applied to improve the footprint shape. Eventually, the roof shape is extracted using RANSAC.

\subsection{Selection of building candidates}

In a first step, the DSM was normalized based on morphological grayscale reconstruction (Vincent, 1993). White morphological reconstruction can extract off-terrain objects and removes smallscale variations from a dark background. We will subsequently call this product normalized DSM (or nDSM).

By applying a binary height threshold of $2 \mathrm{~m}$ to the nDSM and then eroding it by a disk-shaped structuring element of radius 5 pixels, only larger pixel aggregations were left behind. If the resulting mask then fulfilled an area size threshold of $32 \mathrm{~m}^{2}$ (which 
corresponds to about 4000 pixels at $9 \mathrm{~cm}$ resolution), the remaining regions were considered as building candidates. Every building candidate was then subsetted from both $\mathrm{nDSM}$ and RGB image with a rectangular buffer of 200 pixels around its outermost pixels.

\subsection{LSD algorithm and line segment selection}

The Line Degment Detector (LSD) proposed by von Gioi et al. (2012) identifies line segments by region growing of pixels that display the same brightness gradient and orientation in panchromatic images. Several mechanisms prevent the algorithm from detecting noisy features or growing too large line segments which is not present in the well-established Hough transform line detection algorithm (von Gioi et al., 2010).

Hence, we first transformed the RGB aerial image to a panchromatic version. A first run of the LSD algorithm was used to identify the most dominant orientation of line segments present in the image (Fig. 1). There are two ways to proceed. The most intuitive one is to rotate every line segment according to the dominant orientation to align the bulk part of segments with the coordinate axes. However, if high-level languages like Python are used which utilizes the computational advantage of low-level languages, namely $\mathrm{C}$, in its libraries, it may also be conceivable to rotate the whole image instead and repeat the line segment detection. We therefore chose the second approach. For a better understanding of the proposed approach, Figure 2 describes the footprint detection workflow by using an exemplary building. The rotated image and nDSM subsets are shown in Figure 2a and $2 b$. We accepted that another run of the LSD algorithm on the rotated dataset (Fig. 2c) is likely to produce slightly different results. We made the assumption that most buildings are rectangular which greatly boosts the introduced algorithm and is true for a large fraction of buildings in the study area. By also computing the chi-squared test for homogeneity (p-value set to 0.05 ), any building candidate featuring an equal distribution of line segment orientations was discarded as this strongly indicates either vegetation or non-rectangular building footprints (e.g. circular or elliptical shapes)

To account for the image artifacts initially mentioned, all remaining line segments were checked for their position in the nDSM. If they fell below the set height threshold of $2 \mathrm{~m}$, they were removed. The rest was classified by the height gradients to both

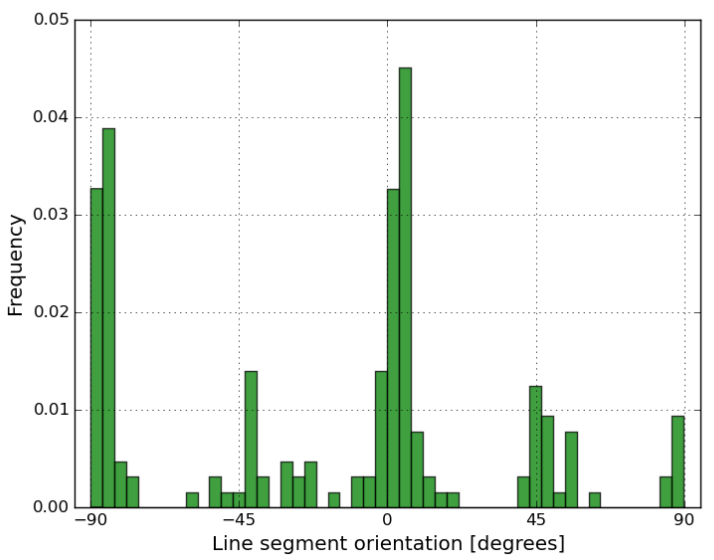

Figure 1: Distribution of line segment angles in a rectangular building (where $0^{\circ}$ corresponds to an horizontal orientation) a)

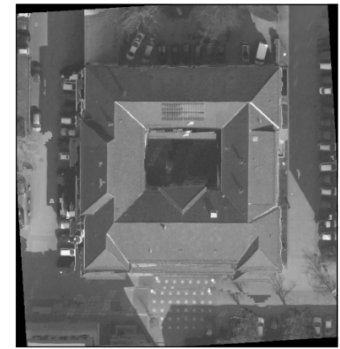

c)

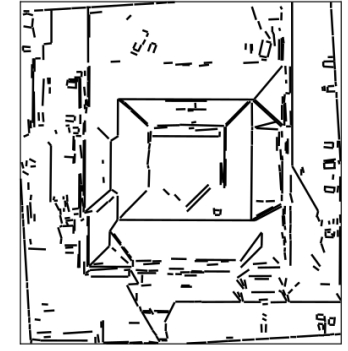

e)

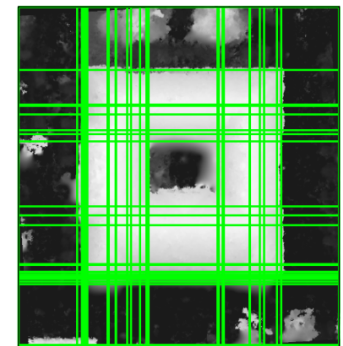

g)

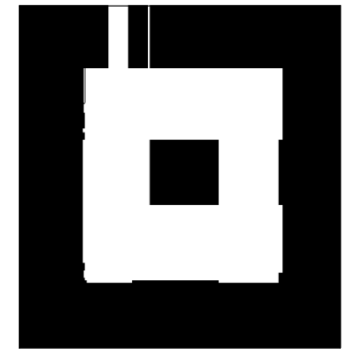

b)

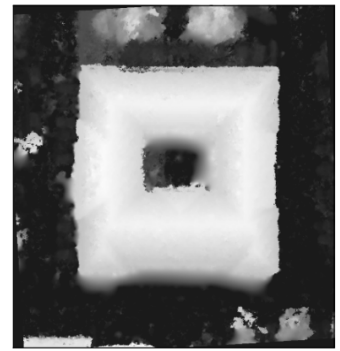

d)
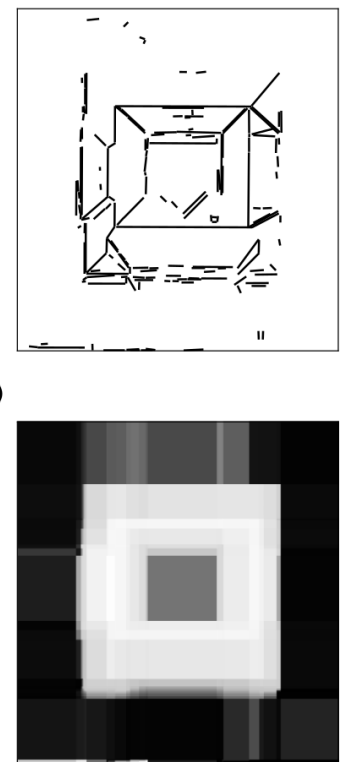

h)

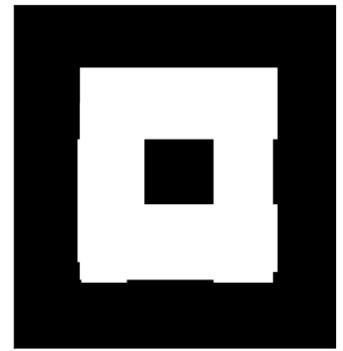

Figure 2: Footprint detection workflow; (a) rotated panchromatic aerial image, (b) normalized DSM (nDSM), (c) detected line segments using the LSD algorithm, (d) line segments fulfilling the gradient criteria, (e) extended line segments that fulfill the orientation criteria, (f) averaged grids, (g) thresholded image filtered by standard deviation, (h) image after morphological filtering

sides of the line, leaving $3 \times 3$ possible combinations (the gradients on each side can be either ascending, descending or horizontal using simple linear regression; Fig. 3). We kept only those line segments that featured differently classified gradients except for line segments with two horizontal gradients above the mentioned height threshold since they may represent important roof architecture features. By doing this, many line segments found in the fuzzy interpolated boundary regions of buildings or their shadows were efficiently removed (Fig. 2d). The interpolated regions do not contain real data. Therefore, we knowingly accepted that this step would also delete line segments close to actual underlying boundaries but found where buildings appeared twice in 


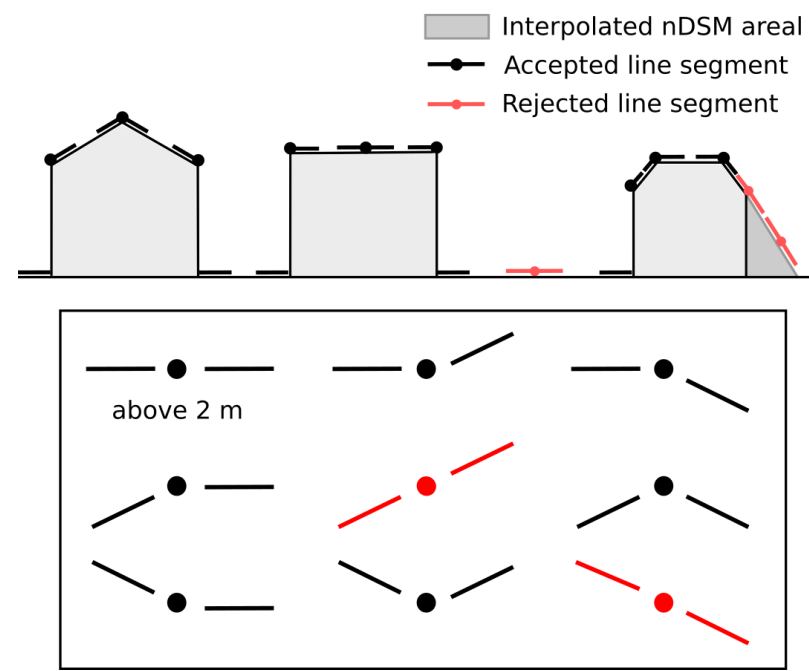

Figure 3: Principle of gradient classification. In the upper crosssection, black/red dots represent line segments while the lines to both sites are their respective gradients. Different gradient combination options are displayed below.

the RGB image. In accordance with the assumption of rectangular buildings, the algorithm eventually selected only those line segments that were aligned with or perpendicularly orientated to the major orientation within a threshold interval of $\pm 5^{\circ}$.

\subsection{Footprint generation and optimization}

The remaining line segments were then rotated to the nearest coordinate axis and extended over the whole image. Since we only took rectangular lines, one of those axes usually lies within $5^{\circ}$ of each line segment. The result is a grid structure segmenting the building in multiple parts (Fig. 2e). This approach is used by a number of authors, including Zebedin et al. (2008) and Grigillo and Kanjir (2012). Non-rectangular grids are equally conceivable and were used by Zebedin et al. (2008). The statistical mean was then derived from the underlying nDSM for every grid (Fig. 2f). We obtain a measure for homogeneity of each grid by means of standard deviation ( $\sigma$ of 3.5) which is useful to filter out vegetation close to buildings, interpolated areas and grids which do not correspond to actual geometries in the scene. By only keeping the largest footprint within the current extent, intersecting extents are less likely to mutually update footprints other than the currently processed one (Fig. 2g).

In order to filter out small-scale variations in the footprint boundary, every candidate underwent morphological erosion with subsequent dilation (Fig. 2h). Considerations on the size of the structuring element are required. If a part of a building or the building as a whole is removed by erosion, it cannot be restored to its initial extent using dilation. If the structuring element is too small, it will not succeed in removing erroneous variations in the boundary region. The choice of an optimal size, therefore, depends on the prevailing type of land-use; industrial zones might require a larger structuring element than residential areas as the general building extent is larger. We chose a $100 \times 100$ pixel square which unlike a disk-shaped structuring element does not round off corners.

The individual building footprints are eventually rotated and reassembled to their initial position in the image.

\subsection{Roof structure reconstruction}

Roof structure reconstruction is a crucial step towards automatic extraction of 3D models from DSM data. Having produced a building footprint in the previous steps, we can generate a 3D point cloud by masking the initial nDSM. As complex building roofs often consist of multiple planar surfaces, we first segmented the image into simpler rectangular features based on the general outline of the building. Kada and McKinley (2009) used the term cell decomposition for a similar approach by referring to Foley et al. (1990). The segmentation of the building into cells is useful as it prevents plane fitting through noncontiguous parts of the building. Segmenting the image based on every variation of the outline is, however, impractical as it creates a large number of very small segments (Fig. 4, left). Instead, we kept only those lines that feature the longest building edge within a lateral buffer of 15 pixels to each side (Fig. 4, right). It is conceivable at this point to apply a further generalization step to the footprint following the cell outline which would make the computation of a generalized building block model with roof (LOD2; Arefi et al. 2008) less complex. This is, however, not implemented here.
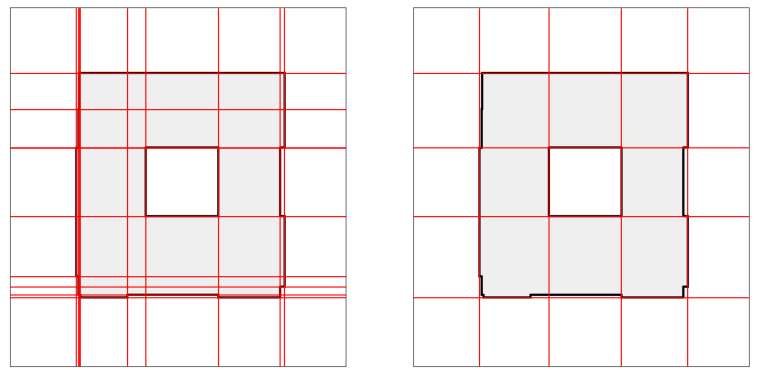

Figure 4: Cell decomposition without (left) and with removal of lines extrapolated from short footprint edges (right)

Random Sample Concensus (RANSAC) was originally conceived for line fitting but can be easily extended to plane fitting problems. We implemented the RANSAC algorithm as described in Yang and Förstner (2010). The basic idea of RANSAC plane fitting is to randomly select three different data points from the point cloud which describe a plane in three-dimensional space. By computing the absolute distance of every other point in the point cloud to this hypothetical plane, we can divide the points into inliers and outliers by applying a distance threshold. This step is repeated for given number of iterations (e.g. 500-1000) to derive a plane hypothesis that comprises a maximum number of inliers. We then deleted those inliers from the point cloud and repeated RANSAC until the number of points is reduced to $10 \%$ of the initial size.

\section{EXPERIMENTS AND DISCUSSION}

\subsection{K data and preprocessing}

In this study, we use an aerial imagery dataset from Karlsruhe, Germany which was acquired with the DLR $3 \mathrm{~K}$ sensor system. The system consists of three commercially available DSLR cameras (one pointing at nadir, two in oblique lateral direction) enabling a FOV of $110^{\circ}$ in across-track direction and $31^{\circ}$ in flight direction (Kurz et al., 2012). The dataset was taken on 20th March 2015 at a flight height of 600 meter above ground level from a Cessna aircraft and consists of altogether $3 \times 750$ images from 10 flight strips covering some relevant parts of the $13 \times 15$ 
$\mathrm{km}$ city area of Karlsruhe. The overlap in flight direction is $80 \%$, whereas a side overlap exists only in regions, when flight strips are parallel or crossing. Thus, occluded regions appear quite often in across flight direction, e.g. behind buildings, as these regions are not covered by the image dataset.
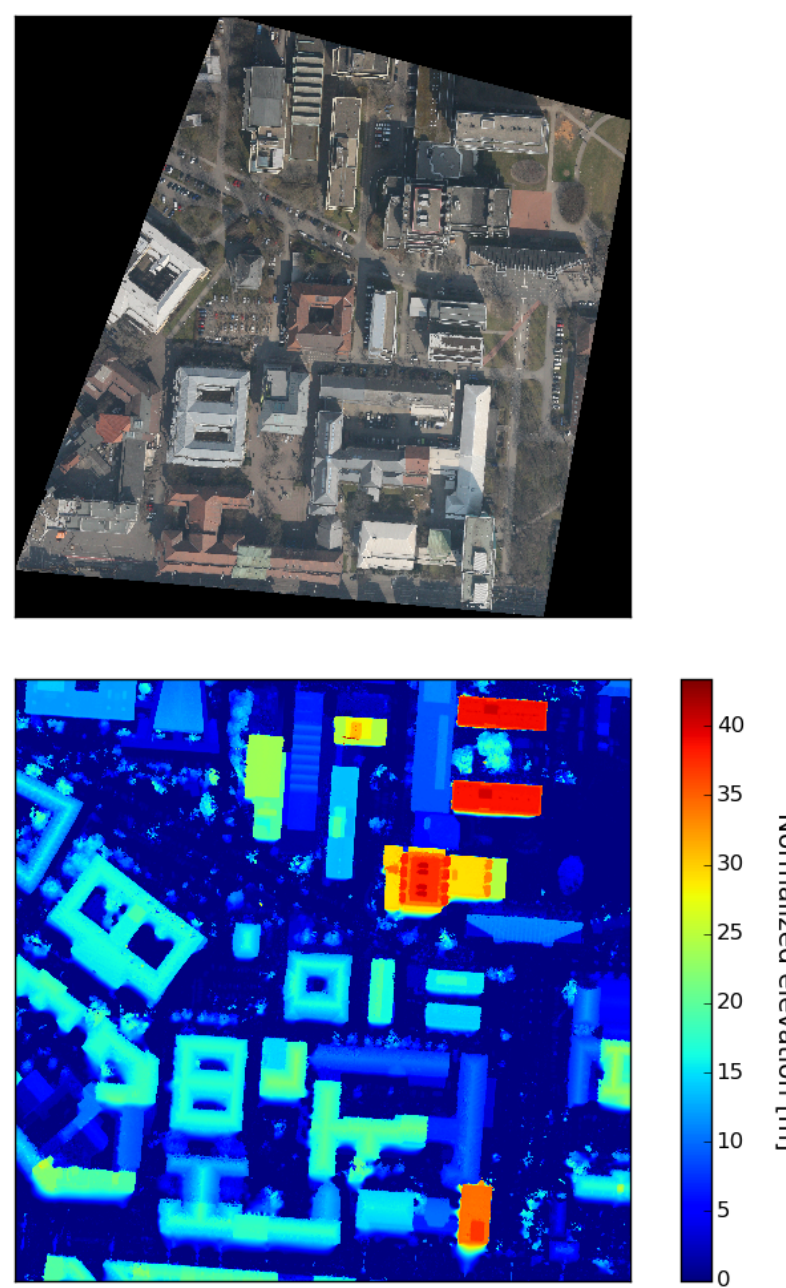

Figure 5: Input data: RGB image (above) and nDSM (below)

A high resolution DSM was derived based on the semi-global matching algorithm and taking into account multiple views with occluded regions being interpolated (d'Angelo and Reinartz, 2011). Based on the GSD of $9 \mathrm{~cm}$ from the imagery, a DSM resampled to $9 \mathrm{~cm}$ resolution was processed for all covered regions in the city of Karlsruhe. The exterior and interior orientations of all images were estimated by a bundle adjustment using the GNSS/IMU measurements. For this process, no ground based pass information was required. After outlier detection and smoothing of the DSM, all images were orthorectified using the derived DSM. In this study, a simple orthorectification process without ray-tracing was applied, which resulted in building edges appearing twice in occluded regions. Wherever possible, a neighboring image was used to fill the gap. Additionally, the inclined pseudo-surfaces in occluded regions lead to confusion with real inclined surfaces such as gable roofs or vegetation. It becomes difficult to avoid interpolation in densily built-up environments due to mutual occlusion.

\subsection{Test sites}

The original RGB image - comprising a size of $4469 \times 4371$ pixels - and DSM are shown in Figure 5 and cover parts of the south-

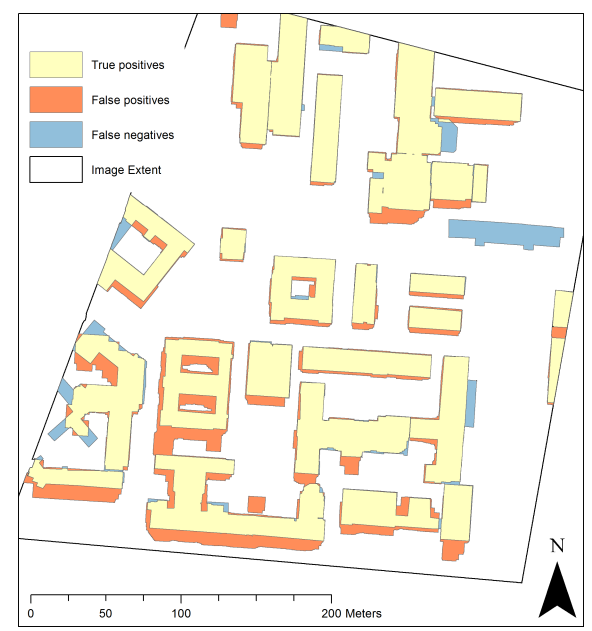

Figure 6: Detection results using our approach

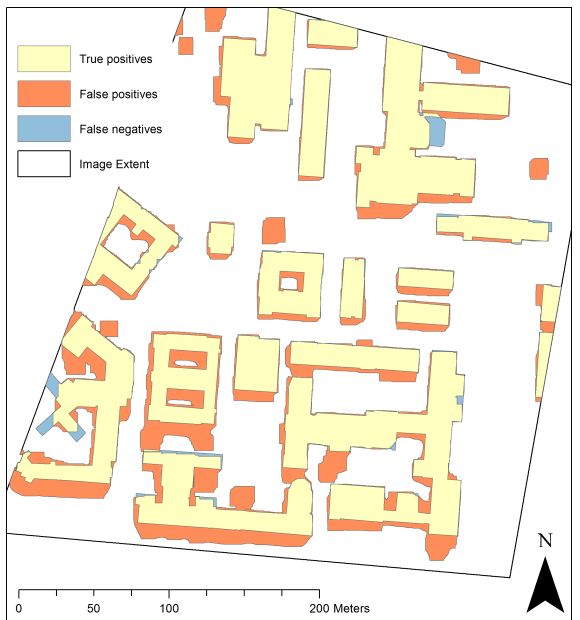

Figure 7: Detection results after thresholding the nDSM and applying a morphological filter

\begin{tabular}{lccc}
\hline & \multicolumn{2}{c}{ Reference Data } \\
\hline & & Building & No building \\
\hline \multirow{2}{*}{ Thresholding } & Building & $30.02 \%$ & $10.81 \%$ \\
& No building & $0.67 \%$ & $58.51 \%$ \\
\hline \multirow{2}{*}{ Overall accuracy (TP + TN) } & $88.53 \%$ & \\
\hline \multirow{2}{*}{ Our approach } & Building & $28.53 \%$ & $6.76 \%$ \\
& No building & $2.16 \%$ & $62.55 \%$ \\
\hline Overall accuracy (TP + TN) & $91.08 \%$ & \\
\hline
\end{tabular}

Table 1: Confusion matrices of the results in Fig. 7 and Fig. 6

ern campus of the Karlsruhe Institute of Technology (KIT). The large variety of building sizes and shapes present in the subset is helpful to assess the performance of the algorithm in different urban settings.

\subsection{Results and evaluation}

The final building footprints are shown in Figure 6. We have overlain the results with manually extracted reference data. Therein, the beige color represents true positives, red areas are false positives and blue areas false negatives. For comparison, we created a second map by applying a simple height threshold of $2 \mathrm{~m}$ to the 
(a) nDSM thresholding and subsequent morphological processing

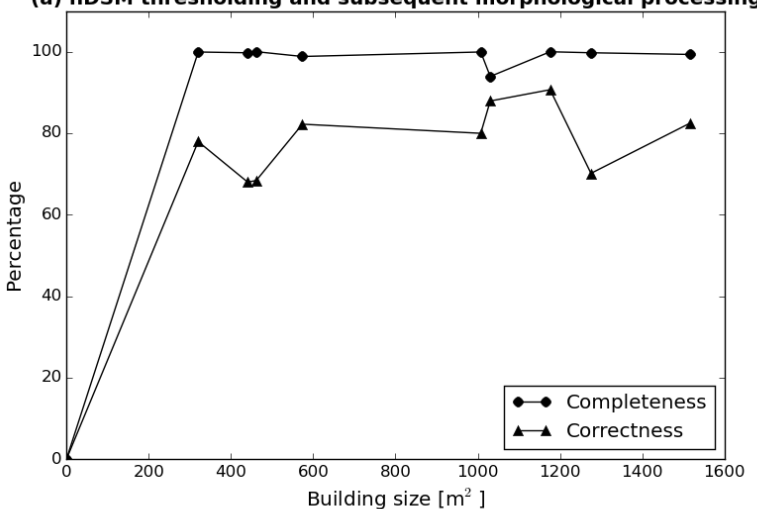

(b) Our approach

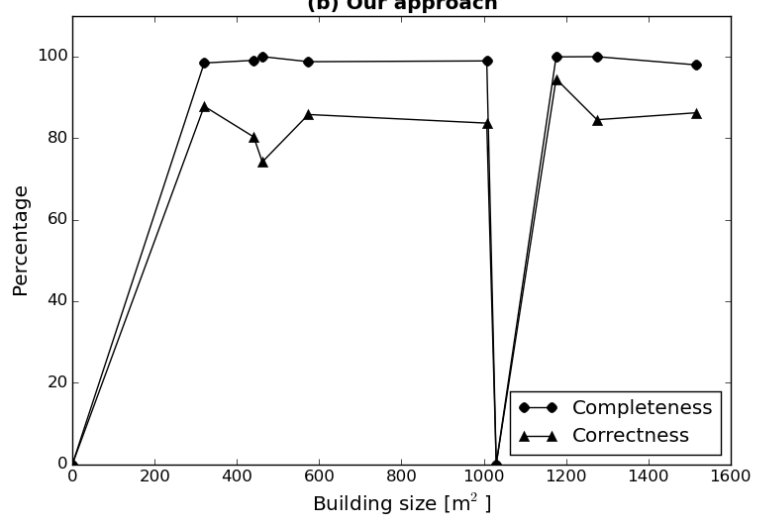

Figure 8: Completeness and correctness of both methods for individual buildings

nDSM and performing the optimization step (Fig. 7). A pixelbased statistical evaluation is recorded in Table 1 .

Overall accuracy as shown in Table 1 may not be representative for the quality of the approach due to the relatively high fraction of areas without buildings. Therefore, we computed the completeness and correctness of the scene as elaborated in Rottensteiner et al. (2005):

$$
\begin{aligned}
& \text { Completeness }=\frac{T P}{(T P+F N)} \\
& \text { Correctness }=\frac{T P}{(T P+F P)}
\end{aligned}
$$

where

$$
\begin{aligned}
& T P:=\text { number of true positives } \\
& F N:=\text { number of false negatives } \\
& F P:=\text { number of false positives }
\end{aligned}
$$

If applied to the thresholded result, we obtain a completeness and correctness value of $97.83 \%$ and $73.53 \%$, respectively. Our approach yields $92.97 \%$ for completeness and $80.84 \%$ for correctness. Nonetheless, Rottensteiner et al. (2005) also postulates the need for individual building assessment. From the subsequent analysis, we excluded regions that were either cut off by the image extent or part of a larger building complex. The latter one is due to the difficult assessment of footprints that were falsely merged or split. The results are shown in Figure 8.
The direct comparison shows the improvement of our approach over the thresholded result. The number of pixels that are false positives considerably decreased (Fig. 6-7, Tab. 1). This is especially the case where vegetation is present. Some exceptions are found where trees of uniform height are closely spaced to buildings. Given the relatively low fraction of vegetation falsely classified as building, separately masking out vegetation by computing the variance of surface normal vectors (Weidner, 1997) did not improve the result but removed many inclined surfaces due the discussed interpolation. Other than that, the algorithm performs reasonably well in environments where buildings are sufficiently spaced to be distinguishable by the preselection process. However, the interpolated areas are still apparent in the resulting image as false positives in the south-facing part of many buildings in Figure 6.

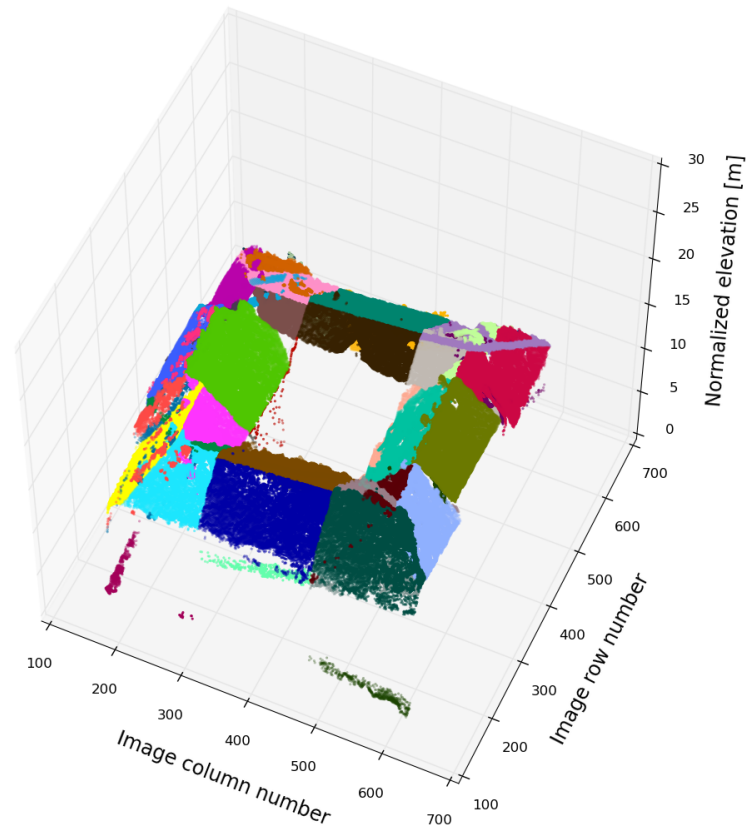

Figure 9: Found plane inliers after applying RANSAC to the building (500 iterations, distance threshold of 0.4 ). The colours imply different planes.

An example for detected planes in a building of average complexity is featured in Figure 9. RANSAC succeeds in detecting the general roof shape of most simple buildings and many more complex shapes. The cell decomposition performed on more complex shapes sometimes does not coincide with the roof architecture leading to some detection errors in edge regions.

\subsection{Discussion}

Both the LSD algorithm (von Gioi et al., 2012) as well as the gradient classification depend on the chosen image extent. Hence, the building in Figure 2 uses a smaller buffer of about 150 pixels by contrast to the same building within the full scene being processed (Fig. 6, center). A single line segment is sufficient to alter the grid structure, thus potentially changing the building footprint. If buildings are not completely covered by the image extent or the edge detection fails to find at least one line segment for each footprint edge, the resulting footprint will be erroneous or disappear completely (Fig. 6, upper right corner; Fig. 8b). This is a common issue with very small buildings. Long edges are more robust to detect as they normally produce longer and more numerous line segments, so that a good boundary approximation can be produced even without every line segment meet- 
ing the criteria set by the algorithm. At the same time, very short edges feature less and shorter line segments implying a higher chance of being filtered out by the orientation criteria. The georeferenced images are partly distorted where height information was too sparse. This contributes to the low line segment density at the edges in Figure 2c-e pointing North and East, respectively. It gets even more complex if the boundary is a low-contrast zone which emphasizes the limitations of the used line segment detection method. Local contrast enhancement could solve some of these issues but it may be more appropriate to utilize the multiperspective information that $3 \mathrm{~K}$ imagery provides. A simple brute force approach could repeat the line detection using different viewing angles and/or individual RGB bands and eventually merge all identified line segments for further processing. An alternative was presented by Zebedin et al. (2008) who instead photogrammetrically derived line segment from mutliple views on the same scene. However, $3 \mathrm{~K}$ data may not have enough view angles for this approach.

Larger building complexes or roofs with regular linear patterns - predominantly saw-tooth and corrogated iron roofs as well as roof-mounted solar arrays - typically result in a very large number of line segments of parallel orientation to the building boundary. In this instance, extended line segments project onto other parts of the building and create polygons that would otherwise not emerge or create small-scale variations in the footprint boundary since this part of the DSM is often fuzzy. This issue is addressed by testing the homogeneity of the grid and by the optimization step.

There is also the issue of the large number of assumptions that enter our model in the form of thresholds. Even though it performed reasonably well on the used dataset, it may be necessary to adjust these parameters depending on the sensor and scene type. This, of course, is far from ideal and we might need to apply machine learning-based techniques in future studies to ensure generalizability of the model.

In comparison to simply thresholding the $\mathrm{nDSM}$ by a $2 \mathrm{~m}$ threshold and applying the same optimization procedure to the full image, the building boundaries in the present approach are more distinct and linear but vegetation is still present in both results. This would cause unwanted geometries when roof detection is applied to the respective areas. Furthermore, the structuring element partly creates a staircase effect if the building boundary is not aligned to the images axes. This is especially emphasized in the thresholded result. Nonetheless, we will need to apply more sophisticated approaches by other authors on $3 \mathrm{~K}$ data in order to obtain more helpful conclusions about the quality of our approach.

\section{CONCLUSION}

We showed that building footprint detection using line segments and a noisy nDSM produce results that can be used for 3D model generation. First, we segmented the image in small processing units and applied the Line Segment Detection algorithm. By successively filtering the resulting line segments, we obtained candidate lines of the building outline. We utilized these lines on the nDSM to create a raw building footprint which was further optimized using morphological filtering. Some false alarms remain if small buildings and densely built-up areas are present which is mainly due to limitations of optical line segment detection and the image quality. Nonetheless, we think it is possible to overcome these problems by testing alternate line detection methods. In future work, we also need to allow non-rectangular footprints and dynamic thresholds.
As for the plane detection, visual evaluation of the obtained planes show a good agreement with the expected roof shape. Logical subsequent steps would include a comparison of faces of neighboring building cells whether they have a similar spatial orientation and to merge them where appropriate. We also need to address the accuracy assessment of the RANSAC results by using ground truth information. Future research may target fully automatic 3D model generation based upon the detected building footprint and roof architecture.

\section{ACKNOWLEDGEMENTS}

The authors would like to thank Tom Kneiphof (University of Bonn) for his helpful remarks on the RANSAC algorithm.

\section{REFERENCES}

Arefi, H. and Reinartz, P., 2013. Building reconstruction using dsm and orthorectified images. Remote Sensing 5(4), pp. 1681-1703.

Arefi, H., Engels, J., Hahn, M. and Mayer, H., 2008. Levels of detail in 3d building reconstruction from lidar data. In: ISPRS Remote Sensing and Spatial Information Sciences, Vol. 37, Part B3b, pp. 485-490.

d'Angelo, P. and Reinartz, P., 2011. Semiglobal matching results on the isprs stereo matching benchmark. In: ISPRS Hannover Workshop 2011: High-Resolution Earth Imaging for Geospatial Information, Vol. 38(4), pp. 79-84.

Foley, J. D., van Dam, A., Feiner, S. K. and Hughes, J. F., 1990. Computer Graphics: Principles and Practice. 2 edn, AddisonWesley.

Grigillo, D. and Kanjir, U., 2012. Urban object extraction from digital surface model and digital aerial images. ISPRS Annals of the Photogrammetry, Remote Sensing and Spatial Information Sciences 1(3), pp. 215-220.

Hermosilla, T., Ruiz, L. A., Recio, J. A. and Estornell, J., 2011 Evaluation of automatic building detection approaches combining high resolution images and lidar data. Remote Sensing 3(6), pp. 1188-1210.

Kada, M. and McKinley, L., 2009. 3d building reconstruction from lidar based on a cell decomposition approach. In: U. Stilla, F. Rottensteiner and N. Paparoditis (eds), CMRT09. IAPRS, Vol. XXXVIII, Part 3/W4, pp. 47-52.

Kurz, F., Türmer, S., Meynberg, O., Rosenbaum, D., Runge, H., Reinartz, P. and Leitloff, J., 2012. Low-cost optical camera systems for real-time mapping applications. PhotogrammetrieFernerkundung-Geoinformation 2012(2), pp. 159-176.

Matikainen, L., Hyyppä, J., Ahokas, E., Markelin, L. and Kaartinen, H., 2010. Automatic detection of buildings and changes in buildings for updating of maps. Remote Sensing 2(5), pp. $1217-1248$

Rottensteiner, F., Trinder, J., Clode, S. and Kubik, K., 2005. Using the dempster-shafer method for the fusion of lidar data and multi-spectral images for building detection. Information $\mathrm{Fu}$ sion 6(4), pp. 283-300.

Shorter, N. and Kasparis, T., 2009. Automatic vegetation identification and building detection from a single nadir aerial image. Remote Sensing 1(4), pp. 731-757. 
Tian, J., Cui, S. and Reinartz, P., 2014. Building change detection based on satellite stereo imagery and digital surface models. IEEE Transactions on Geoscience and Remote Sensing 52(1), pp. 406-417.

Vincent, L., 1993. Morphological grayscale reconstruction in image analysis: applications and efficient algorithms. IEEE Transactions on Image Processing 2(2), pp. 176-201.

von Gioi, R. G., Jakubowicz, J., Morel, J.-M. and Randall, G., 2010. Lsd: A fast line segment detector with a false detection control. IEEE Transactions on Pattern Analysis \& Machine Intelligence 32(4), pp. 722-732.

von Gioi, R. G., Jakubowicz, J., Morel, J.-M. and Randall, G., 2012. Lsd: a line segment detector. Image Processing On Line 2, pp. 35-55.

Wang, O., Lodha, S. K. and Helmbold, D. P., 2006. A bayesian approach to building footprint extraction from aerial lidar data. In: 3D Data Processing, Visualization, and Transmission, Third International Symposium on, IEEE, pp. 192-199.

Weidner, U., 1997. Digital surface models for building extraction. In: Automatic Extraction of Man-Made Objects from Aerial and Space Images (II), Springer, pp. 193-202.

Yang, M. Y. and Förstner, W., 2010. Plane detection in point cloud data. In: Proceedings of the 2 nd international conference on machine control guidance, Bonn, pp. 95-104.

Zebedin, L., Bauer, J., Karner, K. and Bischof, H., 2008. Fusion of feature-and area-based information for urban buildings modeling from aerial imagery. In: Computer Vision-ECCV 2008, Springer, pp. 873-886.

Zhang, K., Yan, J. and Chen, S.-C., 2006. Automatic construction of building footprints from airborne lidar data. IEEE Transactions on Geoscience and Remote Sensing 44(9), pp. 25232533. 